\title{
Brauchen wir die Psychiatrie noch als eigenständiges Fach?
}

\author{
Are we Still in Need of Psychiatry as a Special Field Within Medicine?
}

\begin{abstract}
Unlängst forderte ein psychiatrischer Lehrstuhlinhaber eine Neubestimmung dessen, was man künftig unter Psychiatrie zu verstehen habe [1]. Kollege Maier hat die „Vision eines integrierten klinisch-neurowissenschaftlichen Feldes, in dem die Psychiatrie nur ein Schwerpunkt, aber kein eigenständiges Fach mehr sein kann“. Ein Visionär war auch Wilhelm Griesinger, der etwa 150 Jahre zuvor eine fachlich eigenständige Psychiatrie forderte, die sich durch gleichrangige Einbeziehung des körperlichen und des seelischen Aspekts von den anderen medizinischen Fächern, insbesondere der Neurologie, abheben sollte. Eine einseitig hirnzentrierte Psychiatrie, die die „Seelenzustände“ ausklammerte, war für Griesinger ebenso unvorstellbar wie eine Psychiatrie, die sich nicht für das Gehirn interessierte. Es galt ihm übrigens als selbstverständlich, dass sich die „Seelenzustände“ nicht auf Funktionsstörungen des Gehirns reduzieren lassen [2].
\end{abstract}

Die universitäre Psychiatrie tut sich offensichtlich schwer damit, „Seelenzustände“ - also Erlebnisqualitäten, die der Mensch nur subjektiv kundgeben kann - als „wissenschaftliche Tatsachen“ [3] ernst zu nehmen. Erleben wird von vielen Forschern gleichgesetzt mit neurobiochemischen Vorgängen. So gesehen sind Psychosen nichts weiter als Störungen des Hirnchemismus. Damit aber verschwindet die Person des Patienten aus dem Gesichtsfeld. Für die meisten unserer heutigen Neurowissenschaftler ist es nicht eine Person, die erlebt, bzw. sich verhält, sondern ein Gehirn (z.B. [4-6]). Das Gehirn nimmt als fühlendes, denkendes, handelndes Agens die Stelle der Person ein. Dann ist es nur folgerichtig, psychiatrische Krankheiten als neurologische Krankheiten und die Psychiatrie als neurologische Subdisziplin zu sehen. Aus dem Psychiater wurde in den USA bereits der „Clinical Neuroscientist“ oder der „Clinical Psychopharmacologist“. Wenn man aber den Patienten im Namen des szientistischen Objektivitätspostulats auf sein Hirn reduziert, dann hat das konsequenterweise auch für die Arztperson zu gelten. Zuzulassen wären nur noch Gehirne - das des Patienten auf der einen und das des Arztes auf der anderen Seite. Was sich zwischen Arzt und Patient abspielt, wäre als interaktive Neurophysiologie in einer noch zu konzipierenden Fachsprache zu beschreiben. Dass das SubjektivGeistige von der modernen neurowissenschaftlichen Forschung ausgeblendet wird, zeigen die nicht enden wollenden Diskussionen um den „Freien Willen“ (z.B. [7-9]). Griesingers Kritik am „platten und seichten Materialismus, der alles, was nicht mit Händen zu greifen ist für nicht existent hält“ [2], erweist sich als unverändert aktuell.

Ihre eher zögerliche Aufnahme in die medizinischen Fakultäten verdankt die Psychiatrie Hirnpathologen, die die Ursache von Verhaltensauffälligkeiten in feingeweblichen Veränderungen von Leichengehirnen suchten. Die „Klinische Hirnpathologie“ galt als Bindeglied zwischen den morphologisch klar definierten neurologischen Krankheiten und den histologisch mysteriösen Geisteskrankheiten. Um die Wende zum 20. Jahrhundert setzte mit Emil Kraepelin (1856-1926) eine Entwicklung ein, durch die die Psychiatrie ein eigenständiges Profil gewann. Obgleich dem mechanistischen Denken seiner Zeit verpflichtet, erschien Kraepelin auch das, was die Patienten bewegte als wissenschaftlich bedeutsam. Wenngleich der Amateurbotaniker Kraepelin mit seiner nosologischen Programmatik scheiterte, was er in seiner letzten, kaum beachteten Publikation „Erscheinungsformen des Irreseins“ [10] auch einräumte, hat er doch wie kein anderer das Bild einer autarken Psychiatrie geprägt. 
Institutionell stehen sich heute eine forschungsaktive universitäre „Biologische Psychiatrie“ und eine versorgungsorientierte „Sozialpsychiatrie“ gegenüber. Unabhängig davon ob und wie eine solche Aufspaltung begründbar ist, habe ich mich immer über die Akzeptanz der in den jeweiligen Etikettierungen enthaltenen Widersinnigkeiten gewundert [11]. So müsste unsere somatologisch orientierte „Biologische Psychiatrie“ doch eigentlich „Somatopsychiatrie“ heißen, denn Soma (Körper) ist das Antinom zu Psyche (Seele) und nicht Bios (Leben). Auf der anderen Seite ist "Sozialpsychiatrie“ ein Pleonasmus, denn von seelischer Gesundheit/Krankheit lässt sich sinnvoll überhaupt nur in einem sozialen Bezugssystem sprechen.

Die Wunschvorstellung eines gedeihlichen Miteinanders erwies sich schnell als illusionär. Für den Psychiatrie-Arzt steht eine hilfsbedürftige Person mit ihren konkreten Lebensproblemen im Zentrum. Der Psychiatrie-Forscher interessiert sich demgegenüber für objektiv erhobene Messwerte stärker als für das, was dem Patienten bedeutsam erscheint. Dabei läuft er Gefahr, zu übersehen, dass eine Person primär ein erlebendes Subjekt und erst danach ein physikochemischer Mechanismus, d.h. Objekt ist. Paradoxerweise waren es philosophisch fundierte Physiker wie Bohr [12] oder Heisenberg [13], die nicht müde wurden, auf diesen von den meisten Biowissenschaftlern und Medizinern bis heute nicht zur Kenntnis genommenen Unterschied zwischen der objektiven Wirklichkeit der Physik und unserer Lebenswirklichkeit hinzuweisen.

Lebewesen im Allgemeinen und Menschen im Besonderen lassen sich nur unter einem Doppelaspekt adäquat charakterisieren, nämlich zum einen als Entitäten im Sinne eines zusammengesetzten (Compositum) und zum anderen im Sinne eines unteilbar-integrierten Ganzen (In-dividuum). Wer glaubt, dass sich der letztere Aspekt auf den ersteren zurückführen, eine duale Beschreibung sich also auf eine „eigentliche“ reduzieren lasse, der ignoriert den fundamentalen Unterschied zwischen lebenden Organismen und mechanischen Artefakten. Die Beziehung zwischen den Teilen ist Gegenstand der Physiologie (Funktionsaspekt), die des organismischen Individuums mit seiner Umwelt, Gegenstand von Psychologie/Soziologie (Leistungsaspekt). Für die Medizin und in besonderer Weise die Psychiatrie folgt daraus, dass auch Kranksein prinzipiell unter diesem Doppelaspekt zur Kenntnis zu nehmen ist. Dies gilt für jede Art von Kranksein, vom banalen Knochenbruch bis hin zur bunt schillernden Wahnpsychose. Die Unterschiede liegen lediglich in der jeweiligen, pragmatischen Gesichtspunkten folgenden Gewichtung des einen oder des anderen Aspekts, denn jede Erkrankung ist eine „psychosomatische“. So liegt es auf der Hand, dass das Gelingen einer Operation ungleich mehr vom handwerklichen Geschick als von der Berücksichtigung des psychologisch-personalen Aspekts im OP abhängt. Dieser Aspekt wird erst in der Restitutionsphase größere Bedeutung erlangen. Weitaus kritischer sollten wir diejenigen Fachkollegen sehen, die ihre Patienten vorwiegend oder ausschließlich durch die Brille der Neurochemie sehen. So „bekannte“ erst kürzlich der Direktor eines renommierten Max-Planck-Instituts für Psychiatrie in einem Interview mit einer überregionalen Wochenzeitung [14], dass er sicher ein besserer Chemiker als Psychiater sei. Diese nicht in Zweifel zu ziehende Selbsteinschätzung sollte nun nicht als Ausdruck löblicher Selbstkritik fehlgedeutet werden. Viel nahe liegender ist es, dass hier jenem „Main-Stream“ Tribut gezollt wird, wonach der Beruf des Psychiaters ein Anachronismus sei (s.o.). Im Unterschied zum Psychiatrie-Forscher wird dem Psychiatrie-Arzt immer nur derjenige Kollege als fachkompetent gelten können, dem es gelingt, mit seinen Patienten eine therapeutisch tragfähige kommunikative Basis herzustellen.

Aus der für die gesamte Medizin geltenden Forderung nach einem Aspektdualismus ist abzuleiten, dass „Entweder-oder-Fragestellungen“ durch „Sowohl-als-auch“-Fragestellungen“ zu ersetzen sind. Dementsprechend ist es unzulässig, die Frage aufzuwerfen, ob etwa Hysterie eine Hirnkrankheit oder eine psychologische Krankheit oder ein depressives Syndrom eine biochemische Störung oder eine psychosoziale Störung sei. Derlei nominalistische Definitionen sind genauso verfehlt wie die Frage, ob ein Altarbild ein Kunstgegenstand oder ein sakraler Gegenstand sei.

Neueren Datums ist das Missverständnis, das sich um die Forderung nach einem theoriefreien Beschreiben rankt. Die Psychopathologie wurde vom grünen Tisch der Konsensuskommissionen aus schrittweise durch eine operational definierte Begrifflichkeit ersetzt. In einem nominalistischen Prokrustesbett können wir nur noch das an unseren Patienten zur Kenntnis nehmen, was uns der Merkmalskatalog wahrzunehmen gestattet. Glatzel [15] traf ins Schwarze, als er vor 15 Jahren einem Aufsatz den Titel gab: „Die Abschaffung der Psychopathologie im Namen des Empirismus“. Heute ist die „Biologische Psychiatrie“ vom Mehltau einer terminologischen Abbreviatur überzogen, unter der das personale Individuum unsichtbar geworden ist. Eine unvermeidliche Konsequenz war die Produktion einer Unmenge von Daten, weder der Interpretation fähig noch bedürftig, da in aller Regel nicht reproduzierbar.

Ein besonders lehrreiches Kapitel für Fehlentwicklungen stellt die heute nicht mehr existente psychiatrische EEG-Forschung dar. Theoretische Begründungen schienen entbehrlich. Maßgeblich war allein der naive Glaube, durch Einsatz einer neuartigen Technologie den großen wissenschaftlichen Durchbruch („major break-through“) zu erzielen. Das absehbare Scheitern wurde voraussehbar und ebenfalls ohne Begründung - der Unergiebigkeit des EEG angelastet, das damit auf unabsehbare Zeit als Forschungsinstrument diskreditiert sein dürfte. Kaum 15 Jahre ist es her, dass weite Kreise innerhalb unserer „Biologischen Psychiatrie“ den Ankündigungen gewisser US-amerikanischer „Opinion Leaders" vertrauten, dass es möglich geworden sei, durch Parametrisierung einer einminütigen mehrkanaligen EEG-Ableitung zu DSM-III-R-konformen Diagnosen zu gelangen $[16,17]$. Einwände grundsätzlicher Art gegen eine derartige Eulenspiegelei blieben ebenso unbeachtet wie van Praag [18], der als Rufer in der Wüste seit Jahrzehnten auf das triviale Faktum hinweist, dass die „biologische“ Validierung eines bloßen Namens, wie eben einer DSM-III-Diagnose, einem Wunder gleichkäme. So platzte dann auch besagtes Projekt in zeitlichem Zusammenhang mit der „EEG-Mapping - Blase“ noch ehe es begonnen hatte [19].

Dass man aus früheren Fehlern nichts gelernt zu haben scheint, zeigt der gegenwärtig weltweite Boom der „bildgebenden Verfahren“ in der psychiatrischen Forschung: „Mittlerweile gibt es Untersuchungen mit der funktionellen Kernspintomographie und 
Positronenemissionstomographie zu praktisch allen psychischen Erkrankungen" und weiter:

„In den wenigen Jahren ihrer Existenz hat die funktionelle Bildgebung das Verständnis psychopathologischer Phänomene revolutionär verändert.“ Als Ziel gilt die: „vollständige Kartierung psychopathologischer Symptome und kognitiv emotionaler Dysfunktionen“ $[20]$.

Solche von keinerlei kritischer Selbstreflexion getrübte Vollmundigkeit erinnert an die PR-Kampagne des Pleitiers Craig Venter im Zusammenhang mit dem „Human Genom Project“.

Die Verheißungen des modischen Forschungsparadigmas „Neuroimaging" sind ebenso abwegig wie eine psychiatrische Diagnostik aus dem EEG.

Der Kulturkritiker Neil Postman sah in der modernen „Herrschaft des Bildes“ den Ausdruck einer Infantilisierung bzw. geistigen Rückentwicklung unserer Gesellschaft, von der die Psychiatrie ja einen Teilbereich repräsentiert. Auf die Psychiatrie übertragen ließe sich die zunehmende Monopolisierung der Forschung durch das Bild als ein regressives Entdifferenzierungsphänomen bezeichnen, Rückenwind für alle, die die fachliche Eigenständigkeit der Psychiatrie ohnehin für einen Anachronismus halten.

Wenn uns der Weiterbestand der Psychiatrie als eines im Patienteninteresse liegenden eigenständigen Faches wirklich angelegen ist, dann sind wir gehalten, uns rechtzeitig zu Wort zu melden. Dies umso mehr, als es heute gerade die patientenfernen Psychiatrie-Forscher sind, die sich mit dem schon von Griesinger beklagten Zeitgeist eines „platten und seichten Materialismus“ verbünden, anstatt diesem durch eigene Denkanstrengung Paroli zu bieten.

\section{Literatur}

${ }^{1}$ Maier W. Leserbrief zum Beitrag von H. Häfner Psychiatrie als Beruf. Nervenarzt 2002; 73: 96 - 99

${ }^{2}$ Griesinger W. Die Pathologie und Therapie der psychischen Krankheiten. Zweite umgearbeitete und sehr vermehrte Auflage. Stuttgart: Krabbe, 1861

${ }^{3}$ Fleck L. Entstehung und Entwicklung einer wissenschaftlichen Tatsache (1935), Neuauflage. Frankfurt a. M.: Suhrkamp, 1994

${ }^{4}$ Roth G. Aus der Sicht des Gehirns. Frankfurt a. M.: Suhrkamp, 2003

${ }^{5}$ Singer W. Ein neues Menschenbild? Gespräche über Hirnforschung. Frankfurt a. M.: Suhrkamp, 2003

${ }^{6}$ Singer W. Selbsterfahrung und neurobiologische Fremdbeschreibung. D Z Phil 2004; 5: 230-237

${ }^{7}$ Elger $C$ et al. Das Manifest. Elf führende Neurowissenschaftler über Gegenwart und Zukunft der Hirnforschung. 2004 http://www.gehirn undgeist.de/blatt/det_gg_manifest

8 Bock T et al. „Nur ein Scheinproblem“. Zu den erkenntnistheoretischen Prämissen der Naturwissenschaften. 2005 http://www.gehirnund geist.de/gehirn_geist/scheinproblem.doc

9 Ulrich G. Das epistemologische Problem in den Lebens-und Neurowissenschaften. Voraussetzungen einer integralen Anthropologie. Wissenschaftler und Verantwortung (im Druck); nach einem Vortrag bei der Tagung der Katholischen Akademie Hamburg zum Thema „Gehirn und Geist“", 14./15.10.2005

${ }^{10}$ Kraepelin E. Die Erscheinungsformen des Irreseins. Z ges Neurol Psychiat 1920; 62: 1-29

${ }^{11}$ Ulrich G. Biomedizin - die Folgenschweren Wandlungen des Biologiebegriffs. Stuttgart: Schattauer, 1997

12 Bohr N. Atomphysik und menschliche Erkenntnis. Braunschweig: Vieweg \& Sohn, 1958

${ }^{13}$ Heisenberg W. Wandlungen in den Grundlagen der Naturwissenschaften. 3. Aufl. Leipzig: Hirzel, 1942

${ }^{14}$ Franz A. Chemie für die Seele. 2005 http://www.zeit.de/2005/41PHolsboer?page=all

15 Glatzel J. Die Abschaffung der Psychopathologie im Namen des Empirismus. Nervenarzt 1990; 61: 276-280

${ }^{16}$ John ER, Prichep LS, Fridman J et al. Neurometrics: Computer assisted differential diagnosis of brain dysfunctions. Science 1988; 293: 162 169

17 John ER, Prichep LS, Almas M. Subtyping of psychiatric patients by cluster analysis of QEEG. Brain Topog 1992; 4: 321 - 326

18 Praag $\mathrm{H}$ van. Moving ahead yet falling behind. Neuropsychobiology 1989; 22: 181 - 193

19 John ER. An International Collaborative Study of Biological Subtyping -first draft; (It. Verteiler unter Beteiligung der Psychiatrischen Universitätskliniken Berlin, Düsseldorf, München, Magdeburg, Bonn, Gießen, Frankfurt, Göttingen, Würzburg, Wien und London). 1994

${ }^{20}$ Kircher T, Schneider F, Sauer H et al. Funktionelle Bildgebung am Beispiel der Schizophrenie. Dtsch Ärztebl 2004; 101 (Heft 27): A 1975 1980 